\title{
Relative Ability of Wedge-Coupled Piezoelectric and Meander Coil EMAT Probes to Generate Single-Mode Lamb Waves
}

\author{
Pouyan Khalili ${ }^{\circledR}$ and Peter Cawley
}

\begin{abstract}
Ultrasonic guided waves are used extensively when checking for defects in petrochemical and other industries and are mostly generated using piezoelectric transducers on an angled wedge or electromagnetic acoustic transducers (EMATs) in different configurations. Low-frequency inspection allows for long-distance propagation, but it is best suited for detecting relatively large defects, while at higher frequencies, the presence of multiple wave modes limit defect detectability, so achieving practical single Lamb mode excitation via careful transduction is very beneficial. This paper investigates the relative ability of angled piezoelectric and meander coil EMAT probes to produce single-mode transduction in the medium ( 1-5 MHz-mm) and high (>5 MHz-mm) frequency-thickness regions of the dispersion curves. The nature of each transducer is studied analytically by simulating the corresponding surface forces, followed by the use of a Fourier transform in time and space (2-D fast Fourier transform) to highlight the excitation region in the wavenumber-frequency space. With angled wedge excitation there is a linear relationship between the excitation frequency and the wavenumber which means that the excitation tends to track typical dispersion curves, allowing for easier pure mode generation. In contrast, the EMAT controls frequency and wavenumber separately which makes it more difficult to generate a pure mode when dispersion curves are close together; however, by narrowing the frequency bandwidth via a large number of cycles in the excitation signal, pure mode generation via an EMAT was shown to be possible even in areas of closely spaced modes. As example cases, analytical results, backed up by experiments, showed that signals dominated by the A0 mode at $1.5 \mathrm{MHz}-\mathrm{mm}$ and also the A1 mode at $18 \mathrm{MHz}-\mathrm{mm}$ can be generated with both angled piezoelectric and EMAT probes.
\end{abstract}

Index Terms-Electromagnetic acoustic transducer (EMAT), fast Fourier transform (FFT), guided waves, higher order mode cluster (HOMC), modal decomposition, mode selectivity.

\section{INTRODUCTION}

$\mathbf{I}$ NSPECTION of pipelines for corrosion defects is one of the main problems encountered by the petrochemical and other industries and becomes very difficult when testing at inaccessible regions. Ultrasonic guided wave methods are used extensively to detect the presence of defects within the structure by monitoring reflected and transmitted signals [1]-[13]. The Lamb wave dispersion curves are shown in Fig. 1; here,

Manuscript received September 28, 2017; accepted January 27, 2018. Date of publication February 2, 2018; date of current version March 30, 2018. This work was supported by the U.K. Research Centre in NonDestructive Evaluation (RCNDE) through the Engineering and Physical Sciences Research Council (EPSRC) under Grant EP/L022125/1. (Corresponding author: Pouyan Khalili.)

The authors are with the Department of Mechanical Engineering, Imperial College London, London SW7 2BX, U.K. (e-mail: p.khalili14@imperial.ac.uk; p.cawley@ic.ac.uk).

Digital Object Identifier 10.1109/TUFFC.2018.2800296 the presence of multiple wave modes at different frequencythickness regimes can be seen.

Long-distance propagation is achieved at frequencies below the A1 mode cutoff (up to $\sim 1 \mathrm{MHz}-\mathrm{mm}$ so in a $10-\mathrm{mm}$ wall thickness the frequency is below $100 \mathrm{kHz}$ ), which minimizes the number of the existing modes but due to the low frequency content, this method is best suited for detecting severe defects [1]-[5]. The low-frequency transduction is commonly achieved through an array of point sources which can be done via direct contact piezoelectric transducers (PZT) [6]-[8], ring excitation with electromagnetic acoustic transducers (EMATs) [9]-[11] or magnetostrictive probes [12], [13]. The medium-frequency regime from $1 \mathrm{MHz}-\mathrm{mm}$ to around $5 \mathrm{MHz}-\mathrm{mm}$ gives better sensitivity to smaller defects but the presence of multiple and often dispersive modes limits defect detectability with this method [14]-[21]. Here, the transduction is usually achieved via piezoelectric transducers mounted on an angled wedge [22]-[26] or EMATs [27]-[31]. There has also been interest in guided wave testing over the 1-5 $\mathrm{m}$ range at much higher frequencies ( $>5 \mathrm{MHz}-\mathrm{mm}$ ); Chandrasekaran et al. [32], [37], Satyarnarayan et al. [33], Jayaraman et al. [34], Ratnam et al. [35], and Swaminathan et al. [36] have introduced a technique they call higher order mode cluster (HOMC) that excites higher order Lamb waves at around $20 \mathrm{MHz}-\mathrm{mm}$ which combine to form a nondispersive cluster [32]-[37]. Because of the highfrequency-thickness value, the excited modes exhibit low surface motion which makes the HOMC insensitive to surface features such as supports, weld patches, and T-joints. Recently, Khalili and Cawley [38] have shown that the features of HOMC are essentially those of the A1 mode in this frequencythickness regime. There have also been studies that use a high-frequency Rayleigh wave to test for sharp surface cracks [39]-[41]. The generation of higher order Lamb waves is mostly achieved via piezoelectric transducers mounted on an angled wedge [32]-[34], [36]-[41] with fewer applications of EMATs [35]. A challenge faced by guided wave inspection is the presence of multiple wave modes, particularly in the medium- and high-frequency regimes, so achieving practical single Lamb mode excitation through careful transduction is very beneficial [9], [10], [38], [30]. The excitation of a pure mode can be very difficult at high-frequency-thickness products as the Lamb modes exhibit very similar dispersion characteristics as shown in Fig. 1.

As stated above, guided waves are mostly generated using piezoelectric transducers on an angled wedge and EMATs 

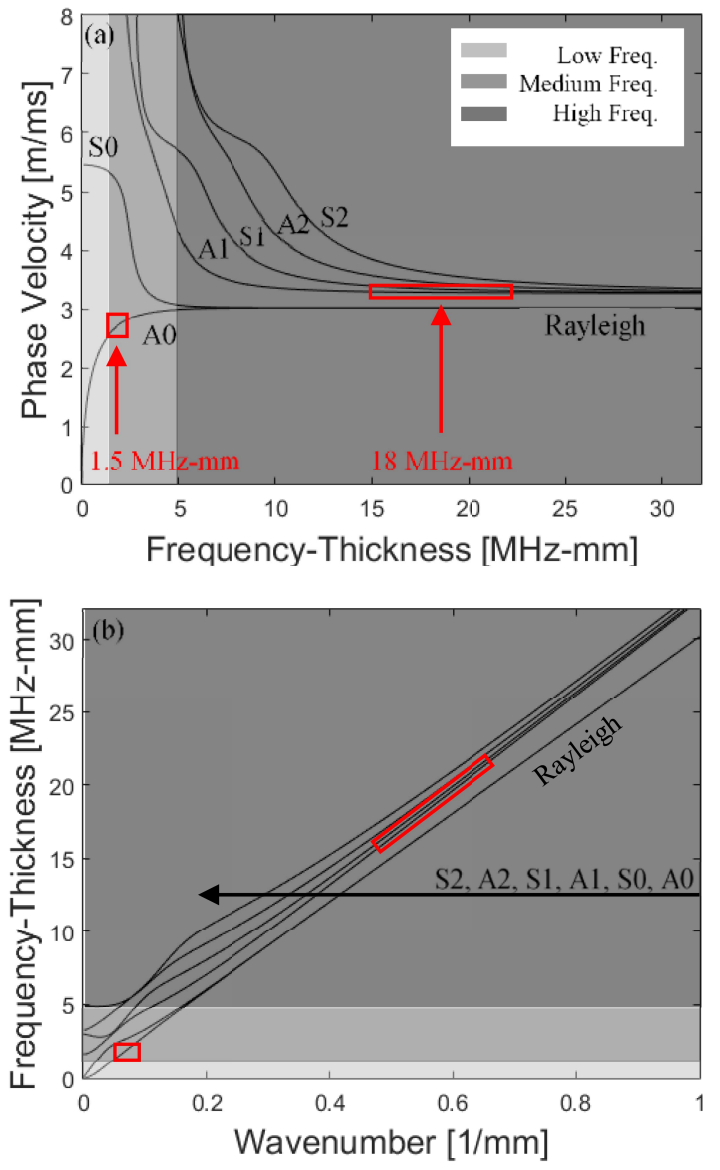

Fig. 1. Lamb wave dispersion curves in steel plate showing (a) phase velocity and (b) wavenumber; generated using DISPERSE [42] software.

in different configurations; however, practical single-mode excitation with each type of transducer is less investigated, particularly at higher frequencies. This paper studies the relative ability of angled PZT and EMAT probes to give pure mode excitation in the medium- and high-frequencythickness regimes. For the medium-frequency case, the A0 mode at $1.5 \mathrm{MHz}-\mathrm{mm}$ was chosen as it offers good application potential such as in structural health monitoring [7], [8], [43]-[45]; the A1 mode at $18 \mathrm{MHz}-\mathrm{mm}$ was studied for the high-frequency case as it provides desirable characteristics for corrosion inspection especially at inaccessible regions [32]-[38].

Section II gives a brief background on the governing physics of piezoelectric transducers on an angled wedge and meander coil EMATs and the design parameters which determine their ability to generate a single mode. Section III then introduces the analytical prediction of the excitation force bandwidths for a given setup through the use of a Fourier transform in time and space [2-D fast Fourier transform (FFT)]. Then these predictions are verified experimentally in Section IV and the findings are summarized in Section V.

\section{BACKGROUND}

Guided waves are often generated by piezoelectric probes where the vibration of the piezoelectric crystal is transmitted to an angled wedge that is connected directly to the surface of the structure through the use of a couplant. This provides strong signal amplitudes and can be implemented with basic equipment but requires direct contact so inconsistent coupling over the footprint, particularly over curved or rough surfaces, is a problem; there are also issues with poor high temperature performance [46] and reverberations within the wedge.

Fig. 2 shows a schematic of a piezoelectric transducer mounted on an angled wedge. In this configuration, the frequency bandwidth is governed by the nature of the excitation signal in time; typically, for guided wave applications, this is specified by the center frequency, the number of cycles used, and the window applied [22], [38]. The wavenumber bandwidth is determined by a combination of the wedge angle $(\theta)$ and the transducer size $(d)$; these parameters dictate the projected excitation length $(L)$ as well as the phasing of the force acting on the surface of the waveguide, as shown in Fig. 2. The key characteristic of the angled wedge assembly is that the frequency and wavenumber bandwidths are interdependent, meaning that if the frequency content $(\omega)$ is changed, it causes the wavelength within the wedge $\left(\lambda_{w}\right)$ and hence the induced wavelength on structure surface $(\lambda)$ to change which consequently results in a change in the number of cycles in space $(L / \lambda)$, thus affecting the wavenumber $(k)$ bandwidth. This characteristic can be formulated as

$$
k_{w}=\frac{\omega}{c_{\mathrm{Lw}}}
$$

where $k_{w}, c_{\mathrm{Lw}}$, and $\omega$ are the wedge wavenumber, the longitudinal wave velocity in the wedge, and the angular frequency, respectively. The wavenumber within the waveguide $(k)$ is

$$
k=\frac{\omega}{c_{p}}
$$

where $c_{p}$ is the phase velocity of the guided wave mode of interest.

As illustrated in Fig. 2(b), the coupling mechanism can be stated

$$
\lambda=\frac{\lambda_{w}}{\sin (\theta)} \text { so } k=k_{w} \sin (\theta) .
$$

From (3) and (1)

$$
k=\frac{\sin (\theta)}{c_{\mathrm{Lw}}} \omega .
$$

Equation (4) shows that the relationship between frequency and wavenumber and can be represented as a straight line with a constant slope of $\left(\sin (\theta) / c_{\mathrm{Lw}}\right)$ in the frequencywavenumber spectrum. Therefore, the frequency-wavenumber relationship of the wedge excitation runs roughly parallel to most regions of the dispersion curves of Fig. 1(b).

Guided wave inspection is often done using a broadly nondispersive mode [15] where excitation at constant phase velocity $c_{p}$ with frequency is achieved with a constant wedge angle. Therefore, for ideal modal selectivity, the wedge angle $(\theta)$ can be calculated

$$
\sin (\theta)=\frac{c_{\mathrm{Lw}}}{c_{p}} .
$$

EMATs on the other hand, rely on the interaction of the dynamic magnetic field of the eddy currents generated by a 


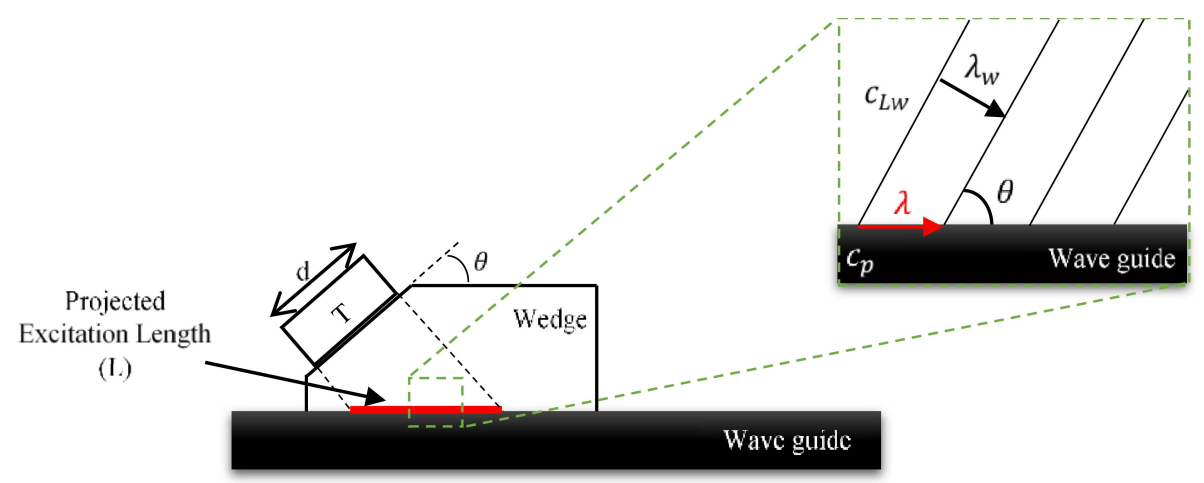

Fig. 2. Transducer schematic of the piezoelectric transducer mounted on an angled wedge. Inset: Relationship between the wavelengths of the compression wave in the wedge and the guided wave in the waveguide.

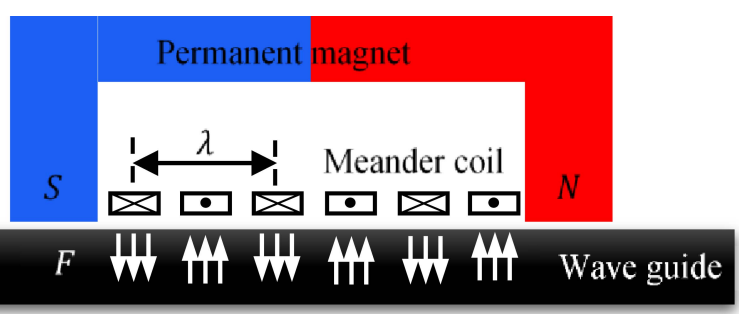

Fig. 3. Transducer schematic of an out-of-plane meander coil EMAT.

coil and the bias magnetic field of a permanent (or electro-) magnet to produce a force resulting in generation of elastic waves in the structure [47]. Hence, they do not require direct contact which eliminates the need for a couplant; this allows transduction over coatings/paint and rough surfaces and also good high-temperature/long-term performance; however, they suffer from poor signal-to-noise ratio (SNR) and often require bulky and expensive electronics.

A typical configuration of a meander coil EMAT is shown in Fig. 3 [47]-[49]. Similar to the piezoelectric probe, the frequency bandwidth is determined by the nature of the excitation signal in time; in contrast, however, the wavenumber bandwidth is dictated by the geometry of the meander coil: the wavelength $(\lambda)$ and the number of cycles in space, as shown in Fig. 3. Therefore, due to the nature of the design, the frequency and wavenumber bandwidths are fully decoupled and independent of each other.

For ideal modal selectivity in the case of a meander coil EMAT, for a given frequency, the wavelength of the coil $(\lambda)$ is chosen according to the wavelength of the mode of interest, while the frequency and wavenumber bandwidths must be carefully calculated in order to minimize the excitation of the unwanted guided modes. In the case of other transducer designs such as periodic permanent magnet EMATs and magnetostrictive devices, the wavenumber bandwidth (and thus selectivity) is also determined by the periodicity of the coil or magnets [50]-[53].

\section{Analytical Prediction of the Excitation FORCE BANDWIDTHS}

The response amplitude of a mode in a waveguide at a particular frequency/wavenumber is directly related to the excitation force acting on the surface at the corresponding frequency/wavenumber [54]. As mentioned above, the frequency and wavenumber bandwidths are dictated by the nature of the transducer design so it is necessary to investigate how to obtain the optimal setup for pure mode excitation in different frequency-thickness regimes for each transducer type. Here a simple analytical model, implemented in the MATLAB software, was employed as it can produce the essential physics while enabling the study of a large number of probe configurations practically. In order to predict the wavenumber and frequency bandwidths of the excitation force, the surface force generated by each transducer was simulated by out-of-plane surface forces followed by the use of a Fourier transform in space and time (2-D FFT). It should be noted that steel was used as the test plate throughout this paper. However, the dispersion relationships for aluminum are similar so the same broad conclusions can also be applied to aluminum (in both cases, it was assumed that the material is isotropic).

In the case of a piezoelectric transducer mounted on an angled wedge, the length of the line force $(L)$ on the surface was obtained from the transducer size $(d)$, and wedge angle $(\theta)$ as shown in Fig. 2; then, the time delay as a function of distance along the surface was calculated according to the angle $(\theta)$ and the acoustic properties of the wedge $\left(c_{\mathrm{Lw}}\right)$ [38]. In this simple model, a collimated beam in the wedge was assumed.

The force pattern for the meander coil EMAT was determined by out-of-plane surface forces with opposite polarity depending on the direction of the acting Lorentz force $(F)$, as shown in Fig. 3; this pattern is repeated according to the wavelength $(\lambda)$ and the number of cycles of the coil in space. Here, for simplicity, the force acting on the surface was assumed to be constant across the width of the coil "finger" [55]. The excitation signal for each coil finger was therefore determined by the applied signal in time and the polarity of the Lorentz force.

\section{A. Medium-Frequency-Thickness Regime}

As an example of the use of the two transducer types in the medium-frequency-thickness regime, this section investigates the relative ability of each transducer type to generate a pure A0 mode at around $1.5 \mathrm{MHz}-\mathrm{mm}(150-\mathrm{Hz}$ center frequency on 

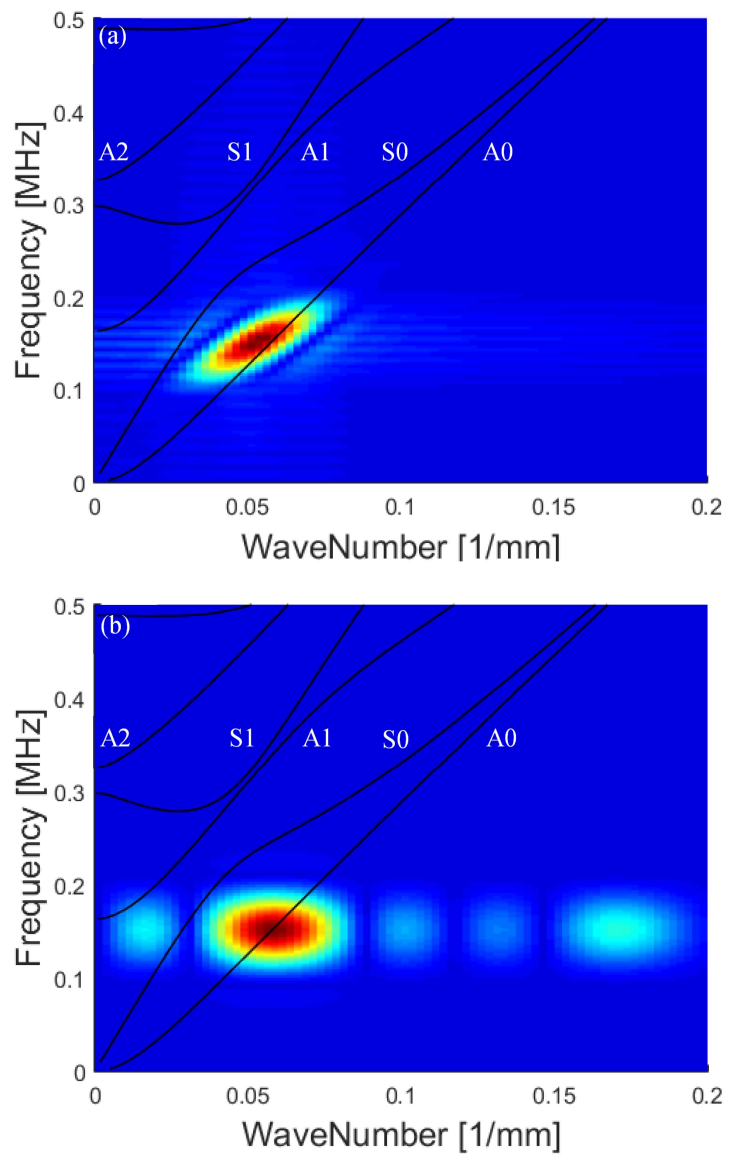

Fig. 4. Two-dimensional FFT color plot [blue (low)-red (high) linear scale] showing the excitation force amplitude in frequency-wavenumber space of (a) 25.4-mm (1 in) compression wave piezoelectric transducer mounted on a $70^{\circ}$ PMMA wedge with a five-cycle Hanning windowed toneburst signal in time at a center frequency of $150 \mathrm{kHz}$. (b) Two-cycle meander coil EMAT with a $17-\mathrm{mm}$ wavelength in space and a five-cycle Hanning windowed toneburst signal in time at a center frequency of $150 \mathrm{kHz}$

a 10-mm thick steel waveguide). Different practical excitation setups were studied for each of the transducer designs through the use of 2-D FFT; for brevity, only the desired excitation configurations are presented in this paper.

Fig. 4(a) shows the excitation force amplitude in the frequency-wavenumber space for a $25.4-\mathrm{mm}$ (1 in) compression wave piezoelectric transducer mounted on a $70^{\circ}$ polymethyl methacrylate (PMMA) $\left(c_{\mathrm{Lw}}=2710 \mathrm{~m} / \mathrm{s}, \rho=\right.$ $1180 \mathrm{~kg} / \mathrm{m}^{3}$ ) wedge where the excitation signal in time consists of a five-cycle Hanning windowed toneburst at a center frequency of $150 \mathrm{kHz}$. In order to demonstrate the effect of a real piezoelectric transducer that does not produce a pure piston-like response, a Hanning window in space was employed; this allows for significant reduction of sidelobes while the wavenumber bandwidth of the central lobe is increased compared to a piston-like response (not shown here) [38]. Here it was found that a 25.4-mm (1 in) piezoelectric transducer is sufficiently large to achieve a narrow enough wavenumber bandwidth to suppress the undesired wave modes. Fig. 4(a) also displays a mismatch between the position of the peak force amplitude and the A0 mode dispersion curve; this is because the phase velocity of the A0 mode at $1.5 \mathrm{MHz}-\mathrm{mm}\left(c_{p}=2610 \mathrm{~m} / \mathrm{s}\right)$ is smaller than the longitudinal

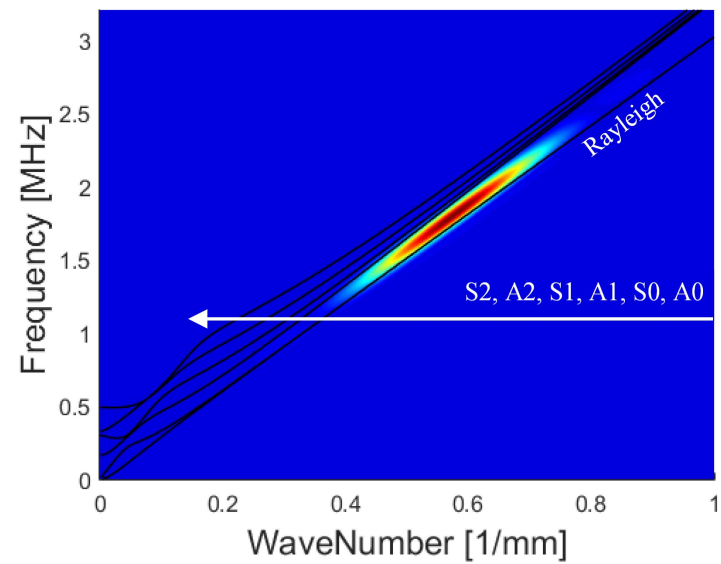

Fig. 5. Two-dimensional FFT color plot [blue (low)-red (high) linear scale] showing the excitation force amplitude in frequency-wavenumber space of a $25.4-\mathrm{mm}$ (1 in) compression wave piezoelectric transducer mounted on a $60^{\circ}$ PMMA wedge with a five-cycle Hanning windowed toneburst signal in time at a center frequency of $1.8 \mathrm{MHz}$.

wave velocity in the PMMA wedge $\left(c_{\mathrm{Lw}}=2710 \mathrm{~m} / \mathrm{s}\right)$ and as demonstrated by (5), ideal selectivity cannot be achieved with PMMA wedge excitation.

Fig. 4(b) illustrates the excitation force amplitude for a two-cycle meander coil EMAT with a $17-\mathrm{mm}$ wavelength in space $(\lambda)$ and a five-cycle Hanning windowed toneburst signal in time at a center frequency of $150 \mathrm{kHz}$. It can be seen that due to the evident separation of the A0 and S0 dispersion curves, the use of a two-cycle coil in space and a fivecycle toneburst signal in time provides sufficiently narrow wavenumber and frequency bandwidths for pure A0 mode excitation. Also, as shown in Fig. 4(b), the sidelobes do not coincide with the undesired modes hence maintaining pure mode excitation.

\section{B. High-Frequency-Thickness Regime}

The example studied in the high-frequency-thickness regime concerns the relative ability of each transducer type to produce pure mode excitation of the A1 mode at $18 \mathrm{MHz}-\mathrm{mm}$ (1.8-MHz center frequency on a 10 -mm-thick steel waveguide). As above, a 2-D FFT is used to calculate the frequency and wavenumber bandwidths in order to find the optimum setup for the best selectivity.

Fig. 5 shows the excitation force amplitude in frequencywavenumber spectrum for a $25.4-\mathrm{mm}$ ( 1 in) compression wave piezoelectric transducer mounted on a $60^{\circ}$ PMMA wedge where the excitation signal in time consists of a five-cycle Hanning windowed toneburst at a center frequency of $1.8 \mathrm{MHz}$; as before, a Hanning window in space was also applied along the projected length $(L)$ in order to mimic the real response of a piezoelectric transducer. As predicted [by (4)], the relationship between the frequency and wavenumber of the wedge excitation is represented as a straight line with a constant slope in the frequency-wavenumber spectrum which, as shown in Fig. 5, runs roughly parallel to the dispersion curves; this means that the frequency bandwidth, which is determined by the signal in time, plays a less crucial role in the selectivity process. Therefore, for a fixed center frequency, the selectivity is dictated by the size of the transducer and the angle of the wedge [38]. 

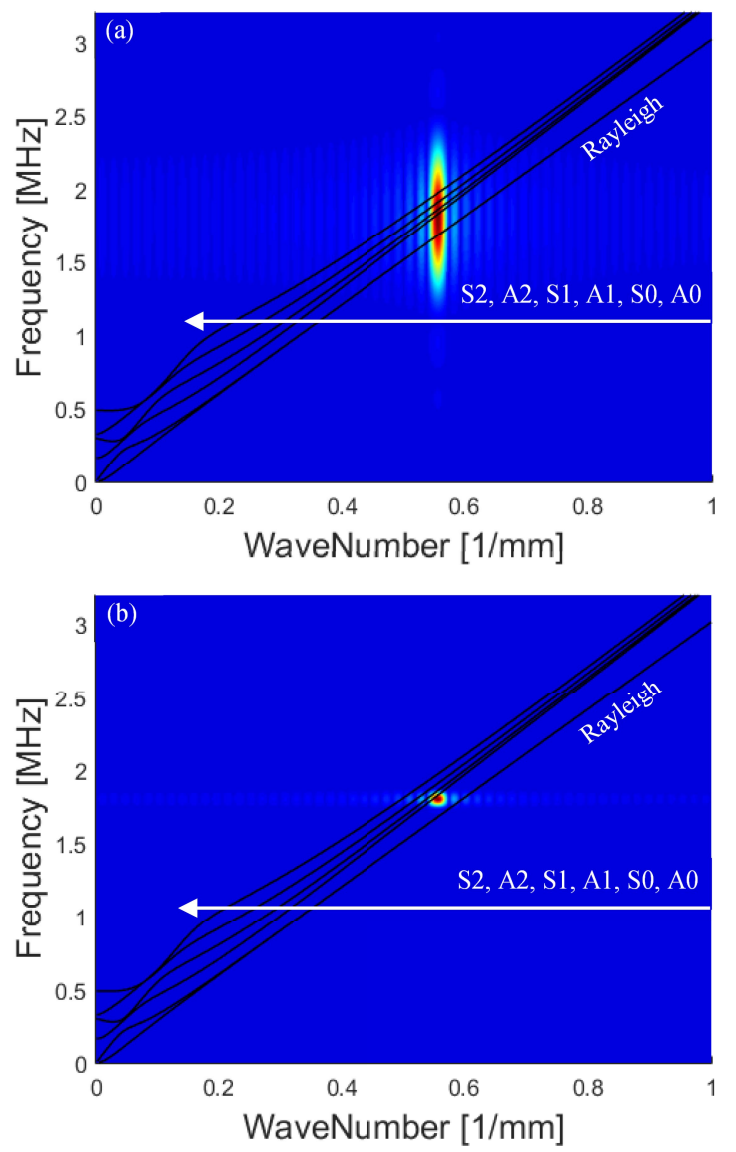

Fig. 6. Two-dimensional FFT color plot [blue (low)-red (high) linear scale] showing the excitation force amplitude in frequency-wavenumber space of a 30-cycle meander coil EMAT with a $1.8-\mathrm{mm}$ wavelength in space and an excitation signal in time of (a) five-cycle Hanning windowed toneburst at a center frequency of $1.8 \mathrm{MHz}$; (b) 50-cycle Hanning windowed toneburst at a center frequency of $1.8 \mathrm{MHz}$.

Fig. 6(a) illustrates the excitation force amplitude for a 30 -cycle meander coil EMAT with a 1.8 -mm wavelength in space $(\lambda)$ and a five-cycle Hanning windowed toneburst signal in time at a center frequency of $1.8 \mathrm{MHz}$. Here, it is clear that the frequency and wavenumber bandwidths are completely independent of each other, which results in a gradient mismatch between the dispersion curves and the excitation force distribution. This means that pure mode excitation of the A1 mode is not possible with a similar signal in time to that used for the wedge excitation. Consequently, in order to suppress the excitation of the other modes compared to the A1 mode, a narrower frequency bandwidth is required.

Fig. 6(b) illustrates the excitation force amplitude for the same meander coil (30-cycle with a $1.8-\mathrm{mm}$ wavelength in space) but with a 50-cycle Hanning windowed toneburst signal in time at a center frequency of $1.8 \mathrm{MHz}$. The significant narrowing of the frequency bandwidth is clearly evident which provides better selectivity of the A1 mode. It should be noted that due to the small wavelength of $\mathrm{A} 1$ at $18 \mathrm{MHz}-$ $\mathrm{mm}(\sim 1.8 \mathrm{~mm})$, the 50 -cycle signal in time maintains good spatial resolution as the total pulsewidth is around $90 \mathrm{~mm}$.

\section{EXPERIMENTAL VALIDATION}

In order to establish the validity of the analytical predictions on the ability of each transducer type to generate

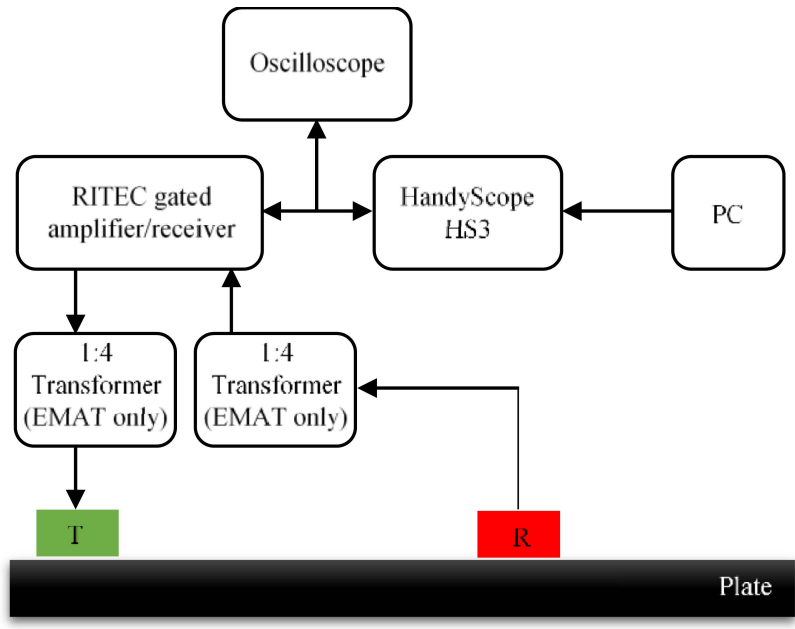

Fig. 7. Schematic of the experimental setup.

pure mode excitation of the A0 and A1 modes, experimental measurements on a steel plate were performed. In the medium-frequency-thickness regime, group velocity measurements were sufficient to identify the propagating mode(s) while for the high-frequency-thickness regime, as with the analytical predictions, 2-D FFT was employed on the surface time traces recorded via a line scan.

\section{A. Experimental Setup}

Fig. 7 illustrates the configuration of the experimental setup used on a 10-mm-thick steel plate. The excitation toneburst was generated via a HandyScope HS3 connected to a computer, the signal being amplified using a RITEC RPR4000 gated amplifier (Ritec Inc., Warwick, RI, USA). In the case of EMAT measurements, transformers were used both for excitation and reception in order to match the relatively low impedance of the EMAT to the relatively high impedance of the amplifier. The output signal from the receiver was then amplified and recorded on a digital oscilloscope.

All the measurements presented here were obtained in pitch-catch configuration. Also in order to ensure sufficient resolution in the 2-D FFT, a line scan was carried out over a 200-mm distance with 0.5 - $\mathrm{mm}$ step intervals.

\section{B. Pure AO Mode Excitation}

Wedge excitation of the lower order Lamb modes has been extensively covered in the literature [22]-[26] so only the EMAT transduction was validated experimentally.

As in the predictions, the EMAT excitation was performed at 150-kHz center frequency via a five-cycle Hanning windowed toneburst which was then amplified and fed into a two-cycle out-of-plane meander coil EMAT with a $17-\mathrm{mm}$ wavelength in space; a similar EMAT was used for reception. To ensure acceptable SNR, the EMATs were electrically shielded and 1000 averages were also performed.

Fig. 8 shows the normalized time trace obtained in pitchcatch configuration over a propagation distance of around $400 \mathrm{~mm}$. Here, the group velocity of the wave packet was obtained through time-of-flight measurements where the peak 


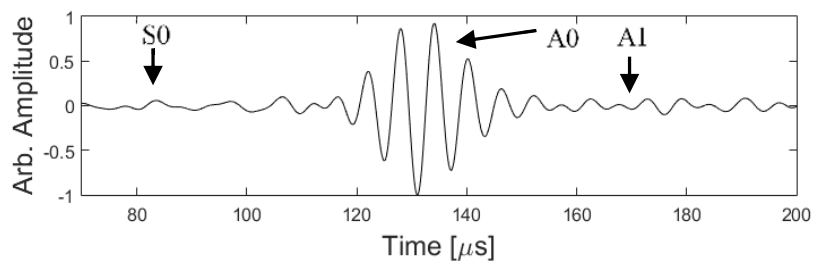

Fig. 8. Time trace obtained in pitch-catch configuration on a 10-mm-thick steel plate using two similar two-cycle meander coil EMATs with a $17-\mathrm{mm}$ wavelength in space over a propagation distance of around $400 \mathrm{~mm}$; the excitation was a five-cycle Hanning windowed toneburst at $150-\mathrm{kHz}$ center frequency.
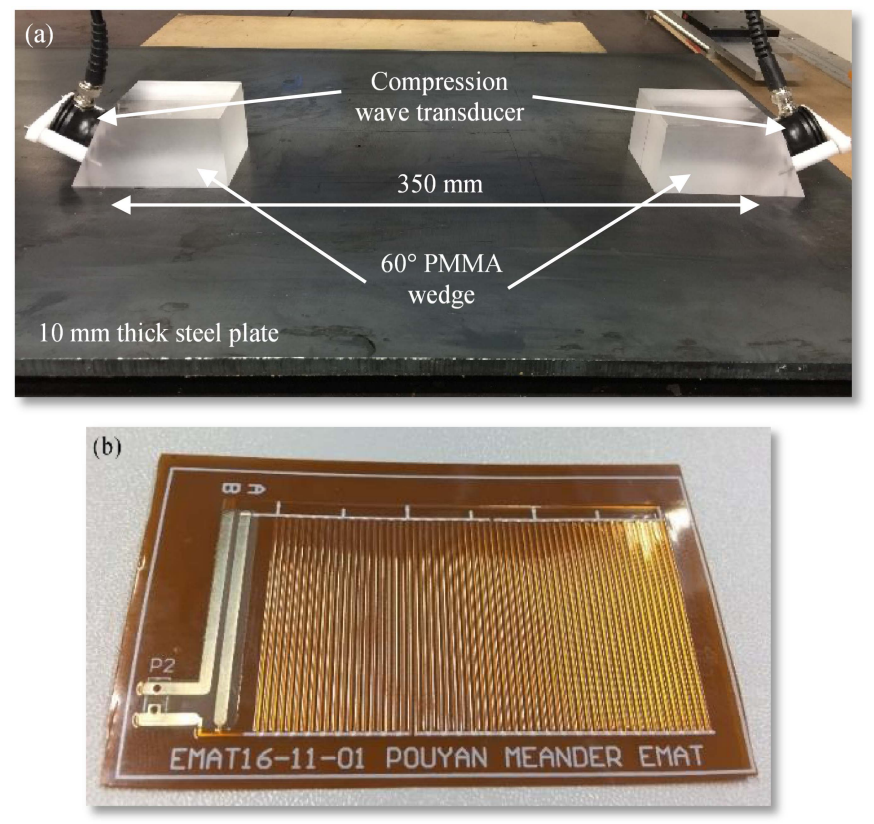

Fig. 9. Picture of (a) 25.4-mm (1 in) diameter compression wave piezoelectric transducer mounted on a $60^{\circ}$ PMMA wedge probes on a $10-\mathrm{mm}$ steel plate; (b) 30-cycle flexible PCB meander coil with a $1.8-\mathrm{mm}$ wavelength in space.

of the wave packet was obtained from a Hilbert transform of the time trace. The wave packet shown in Fig. 8 had a group velocity of $3250 \mathrm{~m} / \mathrm{s}$, which is within $1 \%$ of the group velocity of the A0 mode obtained from the DISPERSE software [42]. The arrival times of the S0 and A1 modes are also displayed in Fig. 8; here, the amplitude ratios of $\mathrm{A} 0 / \mathrm{S} 0$ and $\mathrm{A} 0 / \mathrm{A} 1$ are around $30: 1$ and 18:1, respectively.

\section{Pure Al Mode Excitation}

This section investigates the ability of each transducer type to produce single-mode excitation of the A1 mode at $18 \mathrm{MHz}-\mathrm{mm}$. Due to the possibility of multiple modes and their similar dispersion characteristics, a 2-D FFT was employed for modal decomposition purposes.

Fig. 9(a) shows a pair of 25.4-mm (1 in) diameter 2.25-MHz center frequency piezoelectric compression wave transducers (Panametrics A104S-RB) on a gel-coupled $60^{\circ}$ PMMA wedges on a 10-mm-thick steel plate, the excitation being a five-cycle, $1.8-\mathrm{MHz}$ center frequency Hanning windowed toneburst.

Fig. 9(b) shows the 30-cycle flexible printed circuit board (PCB) meander coil with a $1.8-\mathrm{mm}$ wavelength in
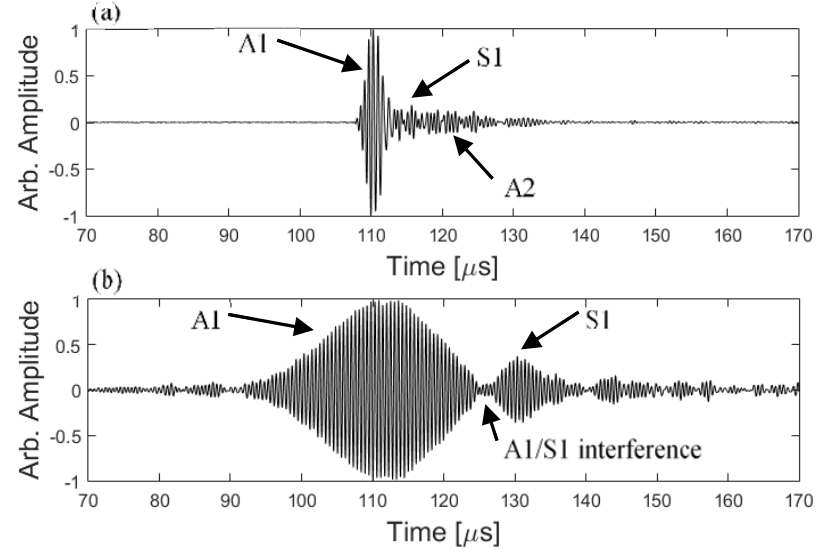

Fig. 10. Time traces obtained in pitch-catch configuration on a 10-mm steel plate with around $350-\mathrm{mm}$ probe separation using (a) two similar probes consisting of a 25.4-mm (1 in) diameter compression wave piezoelectric transducer mounted on a gel-coupled $60^{\circ}$ PMMA wedge where the excitation was performed with a five-cycle Hanning windowed toneburst at $1.8 \mathrm{-MHz}$ center frequency; (b) two similar 30-cycle meander coil EMATs with a $1.8-\mathrm{mm}$ wavelength in space where the excitation was performed with a 50 -cycle Hanning windowed toneburst at $1.8 \mathrm{-MHz}$ center frequency.
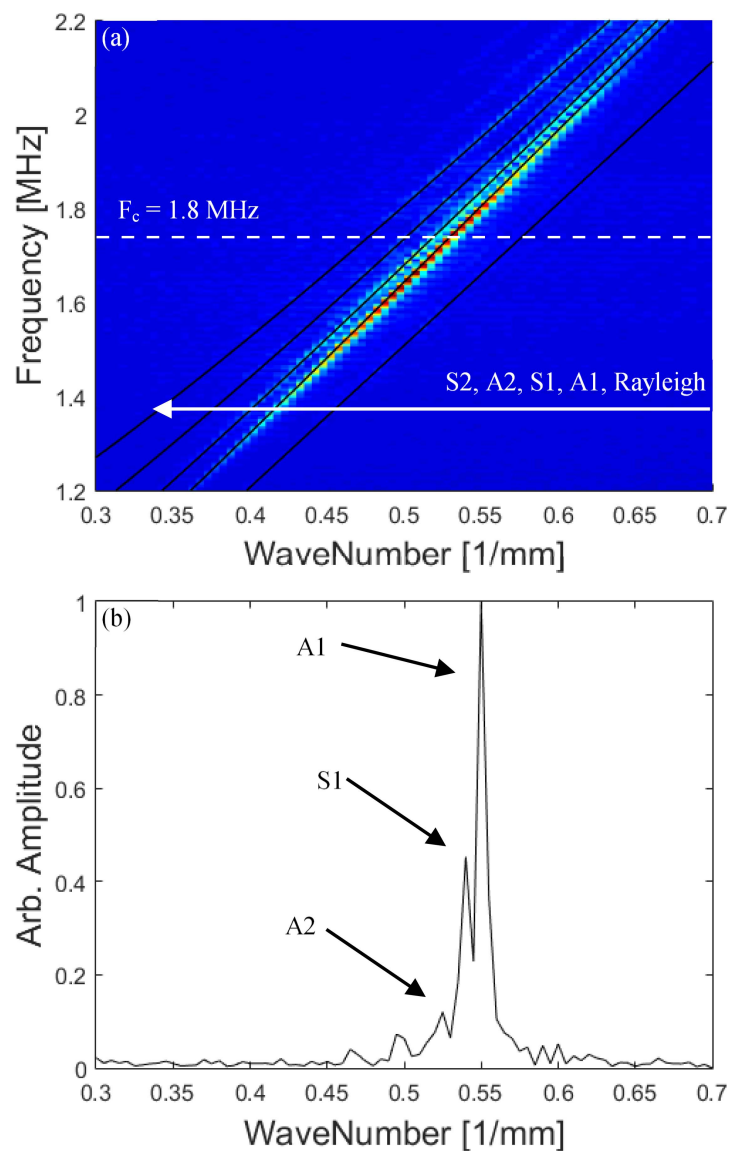

Fig. 11. (a) Two-dimensional FFT color plot [blue (low)-red (high) linear scale] of experimental signal recorded from surface line scan using a 25.4-mm (1 in) diameter compression wave piezoelectric transducer mounted on a gel-coupled $60^{\circ}$ PMMA wedge for a 50-cycle $1.8-\mathrm{MHz}$ center frequency Hanning windowed toneburst excitation signal generated via a similar piezoelectric probe. (b) Amplitude as a function of wavenumber at center frequency $(1.8 \mathrm{MHz})$ obtained from (a).

space used for the EMAT where the excitation was again performed at $1.8 \mathrm{-MHz}$ center frequency but in this case using a 50-cycle Hanning windowed toneburst. Again, the EMATs 

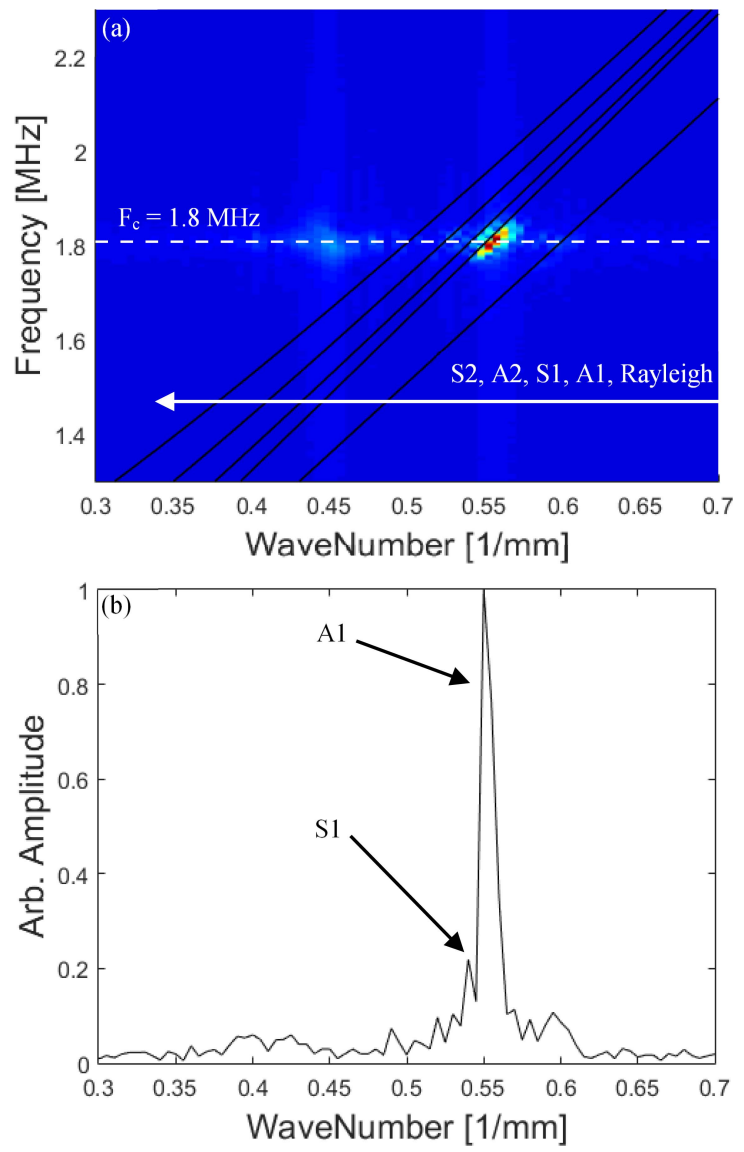

Fig. 12. (a) Two-dimensional FFT color plot [blue (low)-red (high) linear scale] of experimental signal recorded from surface line scan using a 25.4-mm ( 1 in) diameter compression wave piezoelectric transducer mounted on a gel-coupled $60^{\circ} \mathrm{PMMA}$ wedge for a 50 -cycle $1.8-\mathrm{MHz}$ center frequency Hanning windowed toneburst excitation signal generated via a 30-cycle meander coil EMAT. (b) Amplitude as a function of wavenumber at center frequency $(1.8 \mathrm{MHz})$ obtained from (a).

were electrically shielded and 1000 averages were used for each measurement.

Fig. 10(a) shows the time trace obtained using angled wedge excitation in pitch-catch configuration over a $350-\mathrm{mm}$ propagation distance; here the presence of a dominant mode is clearly evident. Fig. 10(b) shows the corresponding time trace for the out-of-plane meander coil EMAT where again the presence of a dominant mode is apparent; in this case the signal duration is much higher because of the use of a 50-cycle excitation signal. For both dominant wave packets displayed in Fig. 10(a) and (b), the group velocity was found to be within $1.5 \%$ of the A1 mode group velocity obtained from the DISPERSE software [42]. It is noteworthy that the relatively short propagation distance (dictated by the experimental setup), at which Fig. 10(a) and (b) time traces were obtained, is not sufficient for complete mode separation as the A1 and S1 modes exhibit similar dispersion characteristics, therefore Fig. 10(a) and (b) do not indicate the true displacement amplitude ratios.

To further confirm the presence of a dominant A1 mode, a 2-D FFT was performed on the time traces obtained from a 200-mm line scan; the EMAT was very difficult to scan accurately at the small intervals necessary for the 2-D FFT, due to its particular design involving the use of strong magnets, so for both excitation transducer types, the time traces were recorded via a $25.4-\mathrm{mm}$ ( 1 in) diameter compression wave piezoelectric transducer mounted on a gel-coupled $60^{\circ}$ PMMA wedge which was moved at intervals of $0.5 \mathrm{~mm}$ in the direction of propagation; here a fine adjustment screw was used to push the probe along guiding tracks and to accurately measure the movement of the probe, a digital Vernier caliper was employed. Fig. 11(a) shows the amplitude in the frequencywavenumber spectrum for the piezoelectric probe, where, as also predicted analytically, A1 is the dominant mode. Fig. 11(b) shows the amplitude as a function of wavenumber at the center frequency $(1.8 \mathrm{MHz})$ obtained from the 2-D FFT of Fig. 11(a); here, the presence of the A1 mode is clearly evident and its amplitude is 2.5 and 10 times higher than that of the S1 and A2 modes, respectively.

Fig. 12(a) shows the linear scale amplitude in the frequency-wavenumber spectrum for the meander coil EMAT transducer, where again, A1 is the dominant mode. Fig. 12(b) shows the amplitude as a function of wavenumber at the center frequency (1.8 MHz) obtained from the 2-D FFT of Fig. 12(a); this shows that the A1 mode is dominant but the precise EMAT-EMAT amplitude ratios cannot be determined because of the use of a wedge receiver rather than the EMAT-EMAT system that was used in the result of Fig. 10(b).

\section{CONCLUSION}

The relative ability of the angled piezoelectric and meander coil EMAT probes to give pure mode excitation in the medium and high-frequency-thickness Lamb mode regimes has been investigated. Analytical predictions of the excitation force amplitude were obtained by simulating the surface forces associated with each transducer followed by the use of a 2-D FFT to highlight the excitation region in wavenumber-frequency space. It was found that with angled wedge excitation there is a linear relationship between the excitation frequency and the wavenumber which means that the excitation tends to track typical dispersion curves, allowing for easier pure mode generation. In contrast, the EMAT controls frequency and wavenumber separately which makes it more difficult to generate a pure mode when dispersion curves are close together.

First, the excitation of the A0 mode at $1.5 \mathrm{MHz}-\mathrm{mm}$ with each transducer type was considered; here, it was found that either of the transducer types operate well due to the wide separation of the dispersion curves. It was shown that good selectivity for the A0 mode can be achieved with a simple two-cycle meander coil EMAT.

Excitation of the A1 mode at $18 \mathrm{MHz}-\mathrm{mm}$ was also studied analytically for each transducer type. In the case of wedge excitation, it was shown that given the small wavelengths at higher frequencies, a typical piezoelectric probe is many wavelengths in diameter, allowing for the excitation to be centered on a narrow wavenumber bandwidth which improves the possibility of pure mode transduction even at a high modal density region of the dispersion curves. As mentioned, due to the nature of the meander coil EMAT, it is more difficult to generate a pure mode at a high modal density region of 
the dispersion curves; however, acceptable selectivity could be achieved by narrowing the frequency bandwidth through increasing the number of cycles in the excitation signal. Given the high frequency, this could be achieved while still maintaining a pulselength below $\sim 30 \mu$ s; this would give adequate spatial resolution in applications where the aim is to inspect over a range of several meters.

Experimental measurements were carried out on a $10-\mathrm{mm}-$ thick steel plate to validate the analytical predictions. The measurements showed good agreement with the predicted results, establishing the possibility of pure mode generation at medium and high-frequency-thickness products with both angled piezoelectric and EMAT probes.

\section{REFERENCES}

[1] D. N. Alleyne, M. J. S. Lowe, and P. Cawley, "The reflection of guided waves from circumferential notches in pipes," J. Appl. Mech., vol. 65, no. 3, pp. 635-641, Sep. 1998.

[2] P. J. Mudge and P. Catton, "Quantification of defect size from long range guided wave ultrasonic tests on pipes," in Proc. AIP Conf., vol. 975. 2008, pp. 147-154.

[3] R. Carandente, J. Ma, and P. Cawley, "The scattering of the fundamental torsional mode from axi-symmetric defects with varying depth profile in pipes," J. Acoust. Soc. Amer., vol. 127, no. 6, pp. 3440-3448, Jun. 2010.

[4] J. L. Rose, M. J. Avioli, P. Mudge, and R. Sanderson, "Guided wave inspection potential of defects in rail," $N D T E$ Int., vol. 37, no. 2, pp. 153-161, Mar. 2004.

[5] W. Mohr and P. Holler, "On inspection of thin-walled tubes for transverse and longitudinal flaws by guided ultrasonic waves," IEEE Trans. Sonics Ultrason., vol. SU-23, no. 5, pp. 369-373, Oct. 1976.

[6] P. Belanger, P. Cawley, and F. Simonetti, "Guided wave diffraction tomography within the born approximation," IEEE Trans. Ultrason., Ferroelect., Freq. Control, vol. 57, no. 6, pp. 1405-1418, Jun. 2010.

[7] T. Clarke, F. Simonetti, S. Rohklin, and P. Cawley, "Development of a low-frequency high purity $\mathrm{A}_{0}$ mode transducer for SHM applications," IEEE Trans. Ultrason., Ferroelect., Freq. Control, vol. 56, no. 7, pp. 1457-1468, Jul. 2009.

[8] N. Hu, T. Shimomukai, H. Fukunaga, and Z. Su, "Damage identification of metallic structures using $\mathrm{A}_{0}$ mode of Lamb waves," Struct. Health Monitor, vol. 7, no. 3, pp. 271-285, Jul. 2008.

[9] M. Seher, P. Huthwaite, M. J. S. Lowe, and P. B. Nagy, "Model-based design of low frequency Lamb wave EMATs for mode selectivity," J. Nondestruct. Eval., vol. 34, no. 3, p. 22, Sep. 2015.

[10] M. Seher, P. Huthwaite, M. Lowe, P. Nagy, and P. Cawley, "Numerical design optimization of an EMAT for $\mathrm{A}_{0}$ Lamb wave generation in steel plates," in Proc. AIP Conf., 2014, vol. 1581. no. 1, pp. 340-347.

[11] B. Herdovics and F. Cegla, "Structural health monitoring using torsional guided wave electromagnetic acoustic transducers," Struct. Health Monitor., vol. 17, no. 1, pp. 24-38, 2016.

[12] Y. Y. Kim, C. I. Park, S. H. Cho, and S. W. Han, "Torsional wave experiments with a new magnetostrictive transducer configuration," J. Acoust. Soc. Amer., vol. 117, no. 6, pp. 3459-3468, Jun. 2005.

[13] S. A. Vinogradov, "Magnetostrictive transducer for torsional guided waves in pipes and plates," Mater. Eval., vol. 67, no. 3, pp. 333-341, 2009.

[14] P. D. Wilcox, M. Lowe, and P. Cawley, "The effect of dispersion on longrange inspection using ultrasonic guided waves," NDT E Int., vol. 34, no. 1, pp. 1-9, 2001.

[15] D. N. Alleyne and P. Cawley, "Optimization of Lamb wave inspection techniques," NDT E Int., vol. 25, no. 1, pp. 11-22, Jan. 1992.

[16] D. N. Alleyne and P. Cawley, "The interaction of Lamb waves with defects," IEEE Trans. Ultrason., Ferroelect., Freq. Control, vol. 39, no. 3, pp. 381-397, May 1992.

[17] J. L. Rose, S. P. Pelts, and M. J. Quarry, "A comb transducer model for guided wave NDE," Ultrasonics, vol. 36, nos. 1-5, pp. 163-169, Feb. 1998

[18] T. R. Hay and J. L. Rose, "Flexible PVDF comb transducers for excitation of axisymmetric guided waves in pipe," Sens. Actuators A, Phys., vol. 100, no. 1, pp. 18-23, Aug. 2002.
[19] M. J. Quarry and J. L. Rose, "Multimode guided wave inspection of piping using comb transducers," Mater. Eval., vol. 57, no. 10, pp. 1089-1090, 1999

[20] P. Wilcox, M. Castaings, R. Monkhouse, P. Cawley, and M. Lowe, "An example of the use of interdigital PVDF transducers to generate and receive a high order Lamb wave mode in a pipe," in Review of Progress in Quantitative Nondestructive Evaluation. Boston, MA, USA: Springer, 1997, pp. 919-926.

[21] J. J. Ditri, J. L. Rose, and G. Chen, "Mode selection criteria for defect detection optimization using Lamb waves," Rev. Prog. Quant. Nondestruct. Eval., vol. 11B, pp. 2109-2115, 1992.

[22] J. L. Rose, Ultrasonic Guided Waves in Solid Media. New York, NY, USA: Cambridge Univ. Press, 2014.

[23] P. D. Wilcox, M. J. S. Lowe, and P. Cawley, "Mode and transducer selection for long range Lamb wave inspection," J. Intell. Mater. Syst. Struct., vol. 12, no. 8, pp. 553-565, 2001.

[24] J. J. Ditri, J. L. Rose, and A. Pilarski, "Generation of guided waves in hollow cylinders by wedge and comb type transducers," Rev. Prog. Quant. Nondestruct. Eval., vol. 12A, pp. 211-218, 1993.

[25] J. L. Rose, "A baseline and vision of ultrasonic guided wave inspection potential," J. Pressure Vessel Technol., vol. 124, no. 3, pp. 273-282, 2002.

[26] J. Li and J. L. Rose, "Excitation and propagation of non-axisymmetric guided waves in a hollow cylinder," J. Acoust. Soc. Amer, vol. 109 no. 2, pp. 457-464, 2001

[27] H.-J. Salzburger, F. Niese, and G. Dobmann, "Emat pipe inspection with guided waves," Welding World, vol. 56, nos. 5-6, pp. 35-43, May 2012.

[28] H. J. Salzburger, "EMAT's and its potential for modern NDE-state of the art and latest applications," in Proc. IEEE Int. Ultrason. Symp., Sep. 2009, pp. 621-628.

[29] R. B. Thompson, G. A. Alers, and M. A. Tennison, "Application of direct electromagnetic Lamb wave generation to gas pipeline inspection," in Proc. Ultrason. Symp., Oct. 1972, pp. 91-94.

[30] Z. Guo, J. D. Achenbach, and S. Krishnaswamy, "EMAT generation and laser detection of single Lamb wave modes," Ultrasonics, vol. 35, no. 6, pp. 423-429, Sep. 1997.

[31] S. Dixon and S. B. Palmer, "Wideband low frequency generation and detection of Lamb and Rayleigh waves using electromagnetic acoustic transducers (EMATs)," Ultrasonics, vol. 42, no. 10, pp. 1129-1136, Aug. 2004.

[32] J. Chandrasekaran, C. V. Krishnamurthy, and K. Balasubramaniam, "Axial higher order modes cluster (A-HOMC) guided wave for pipe inspection," in Proc. AIP Conf., 2010, vol. 1211. no. 1, pp. 161-168.

[33] L. Satyarnarayan, J. Chandrasekaran, B. Maxfield, and K. Balasubramaniam, "Circumferential higher order guided wave modes for the detection and sizing of cracks and pinholes in pipe support regions," NDT E Int., vol. 41, no. 1, pp. 32-43, Jan. 2008.

[34] C. Jayaraman, C. V. Krishnamurthy, and K. Balasubramaniam, "Higher order modes cluster (HOMC) guided waves-A new technique for NDT inspection," in Proc. AIP Conf., 2009, vol. 1096. no. 1, pp. 121-128.

[35] D. Ratnam, K. Balasubramaniam, and B. W. Maxfield, "Generation and detection of higher-order mode clusters of guided waves (HOMC-GW) using meander-coil EMATs," IEEE Trans. Ultrason., Ferroelect., Freq. Control, vol. 59, no. 4, pp. 727-737, Apr. 2012.

[36] A. A. Swaminathan, B. K. Balasubramaniam, C. I. Anto, and K. S. Venkataraman, "Higher order mode cluster (HOMC) guided wave testing of corrosion under pipe supports (CUPS)," Proc. Nat. Seminar Exhib. Non-Destruct. Eval., pp. 224-227, 2011.

[37] J. Chandrasekaran, I. Anto, K. Balasubramaniam, and K. S. Venkataraman, "Higher order modes cluster (HOMC) guided waves for online defect detection in annular plate region of aboveground storage tanks," Insight-Non-Destr. Test. Condition Monitor. vol. 51, no. 11, pp. 606-611, 2009.

[38] P. Khalili and P. Cawley, "Excitation of single-mode Lamb waves at high-frequency-thickness products," IEEE Trans. Ultrason., Ferroelect., Freq. Control, vol. 63, no. 2, pp. 303-312, Feb. 2016.

[39] B. Masserey and P. Fromme, "Fatigue crack growth monitoring using high-frequency guided waves," Struct. Health Monitor., vol. 12, nos. 5-6, pp. 484-493, Sep. 2013

[40] B. Masserey and P. Fromme, "Surface defect detection in stiffened plate structures using Rayleigh-like waves," NDT E Int., vol. 42, no. 6 , pp. 564-572, Sep. 2009.

[41] M. Hirao, H. Fukuoka, and Y. Miura, "Scattering of Rayleigh surface waves by edge cracks: Numerical simulation and experiment," J. Acoust. Soc. Amer., vol. 72, no. 2, pp. 602-606, Aug. 1982. 
[42] B. Pavlakovic, M. Lowe, D. N. Alleyne, and P. Cawley, "Disperse: A general purpose program for creating dispersion curves," Rev. Prog. Quant. Nondestruct. Eval., vol. 16A, pp. 185-192, 1997.

[43] S. Grondel, C. Paget, C. Delebarre, J. Assaad, and K. Levin, "Design of optimal configuration for generating $\mathrm{A}_{0}$ Lamb mode in a composite plate using piezoceramic transducers," J. Acoust. Soc. Amer., vol. 112, no. 1, pp. 84-90, 2002.

[44] B. Xu and V. Giurgiutiu, "Single mode tuning effects on Lamb wave time reversal with piezoelectric wafer active sensors for structural health monitoring," J. Nondestruct. Eval., vol. 26, nos. 2-4, pp. 123-134, Dec. 2007.

[45] T. Clarke, F. Simonetti, S. Rokhlin, and P. Cawley, "Evaluation of the temperature stability of a low-frequency A0 mode transducer developed for SHM applications," in Proc. AIP Conf., vol. 975. 2008, pp. 910-917.

[46] V. A. Attarian, F. B. Cegla, and P. Cawley, "Long-term stability of guided wave structural health monitoring using distributed adhesively bonded piezoelectric transducers," Struct. Health Monitor, vol. 13, no. 3, pp. 265-280, 2014.

[47] K. Mirkhani et al., "Optimal design of EMAT transmitters," NDT E Int., vol. 37, no. 3, pp. 181-193, Apr. 2004.

[48] R. B. Thompson, "Physical principles of measurements with EMAT transducers," in Physical Acoustics. New York, NY, USA: Academic, 1990, pp. 157-200.

[49] R. Thompson, G. Alers, and M. Tennison, "Method for ultrasonic inspection," U.S. Patent 3850028 , Nov. 26, 1974.

[50] C. F. Vasile, "Electromagnetic transducer," U.S. Patent 4127035 , Nov. 28, 1978.

[51] C. F. Vasile, R. B. Thompson, and C. M. Fortunko, "Method and apparatus for efficiently generating elastic waves with a transducer," U.S. Patent 4248092, Feb. 3, 1981.

[52] M. Hirao and H. Ogi, "An SH-wave EMAT technique for gas pipeline inspection," NDT E Int., vol. 32, no. 3, pp. 127-132, Apr. 1999.

[53] R. Ribichini, F. Cegla, P. B. Nagy, and P. Cawley, "Study and comparison of different EMAT configurations for SH wave inspection," IEEE Trans. Ultrason., Ferroelect., Freq. Control, vol. 58, no. 12, pp. 2571-2581, Dec. 2011.

[54] P. Wilcox, "Modeling the excitation of Lamb and SH waves by point and line sources," in Proc. AIP Conf., vol. 700. 2004, pp. 206-213.

[55] R. S. C. Monkhouse, P. D. Wilcox, and P. Cawley, "Flexible interdigital PVDF transducers for the generation of Lamb waves in structures," Ultrasonics, vol. 35, no. 7, pp. 489-498, Nov. 1997.

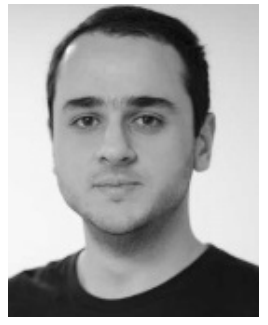

Pouyan Khalili was born in Tehran, Iran, in 1991. He received the M.Eng. (Hons.) degree in mechanical engineering from University College London (UCL), London, U.K., in 2014. He gained interest in the field of non-destructive evaluation (NDE) while studying at UCL, where he worked on numerical modelling of ultrasonic guided waves used to detect impact damage in aerospace composites. He is currently pursuing the Ph.D. degree with the Non-Destructive Testing Group, Imperial College London, London, where he is working on high-frequency guided waves and other methods of corrosion monitoring applied in petrochemical plants.

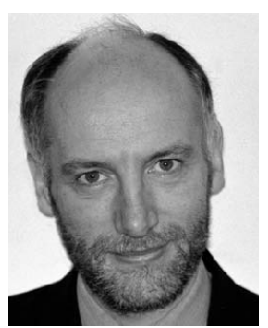

Peter Cawley was born in Sheffield, England, U.K., in 1953. He received the B.Sc. and Ph.D. degrees in mechanical engineering from the University of Bristol, England, in 1975 and 1979, respectively.

He worked in industry from 1979 to 1981 and then joined the Mechanical Engineering Department, Imperial College, London, initially as a Lecturer and then successively Senior Lecturer, Reader, and Professor. He is currently the Head of the Mechanical Engineering Department. He is a current director of two spin-out companies set up to exploit technology developed in his research group (Guided Ultrasonics Ltd., Brentford, U.K., and Permasense Ltd., Crawley, U.K.), and he is a consultant to a variety of industries. He has worked on a wide variety of projects using sonic and ultrasonic methods applied to NDE and he leads the Imperial College NDE group. He is also a leading member of the UK Research Centre for NDE that has its head office at Imperial College. He has authored or co-authored more than 160 refereed journal papers and a similar number of conference papers in this field and holds 4 current patents. 